\title{
Gut feeling from Wuhan: advice for gastrointestinal endoscopy performed during the COVID-19 outbreak
}

Since early 2020, the COVID-19 coronavirus outbreak has led to many cancellations of medical meetings, including live gastrointestinal (Gl) endoscopy courses. Although, the Thai Association of Gastrointestinal Endoscopy (TAGE) annual GI Live Endoscopy Demonstration was able to continue, many overseas faculty members and delegates from China could not participate. TAGE arranged a live-streaming session from Wuhan out of respect for the Chinese endoscopists and their healthcare personnel. The session not only allowed the endoscopy community to show its support for Chinese colleagues who have worked at the frontline, but TAGE and the Gl Live delegates learned about the protective measure taken by Gl endoscopy personnel during the outbreak ( $\triangleright$ Video 1 ). Nevertheless, during a pandemic crisis, all meetings and courses should be canceled.

Although GI endoscopy is not considered a high-risk aerosol-generating procedure like endotracheal intubation [1], a risk of infection to healthcare personnel does exist during close-contact endoscopy. The Wuhan team has recommended many specific measures, including risk stratification of patients ( $\triangleright$ Fig. 1 ) and endoscopy staff ( $\mathbf{F i g . 2}$ ), and three levels of personal protective equipment depending on the infection risk of the patient ( $\vee$ Fig. 3 ).

At the beginning of the COVID-19 outbreak in Wuhan, there was a huge number of potentially infected cases and the number of nucleic acid detection kits, which was the only available diagnostic test, was insufficient; in addition, the false-negative rate of the test was not acceptable. Consequently, computed tomography scan and routine blood testing, both of which are widely available, became the most appropriate screening tests. The idea is to reduce the dispersal of respiratory secretions containing potentially infectious particles.

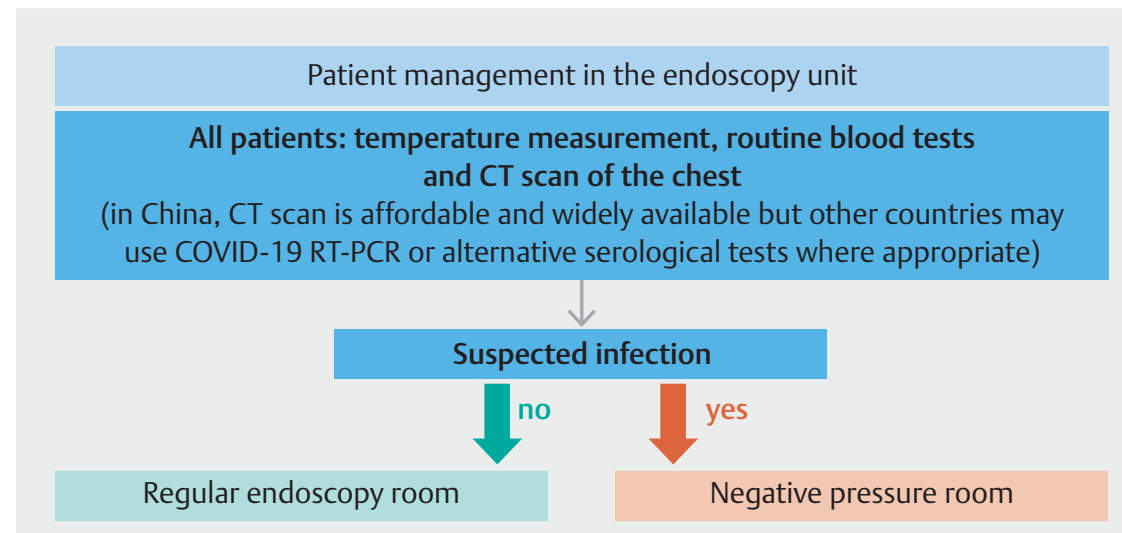

- Fig. 1 Risk stratification of patients using temperature measurement, blood tests, and computed tomography. Patients with suspected infection are moved to a negative pressure environment. RT-PCR, reverse transcription polymerase chain reaction.

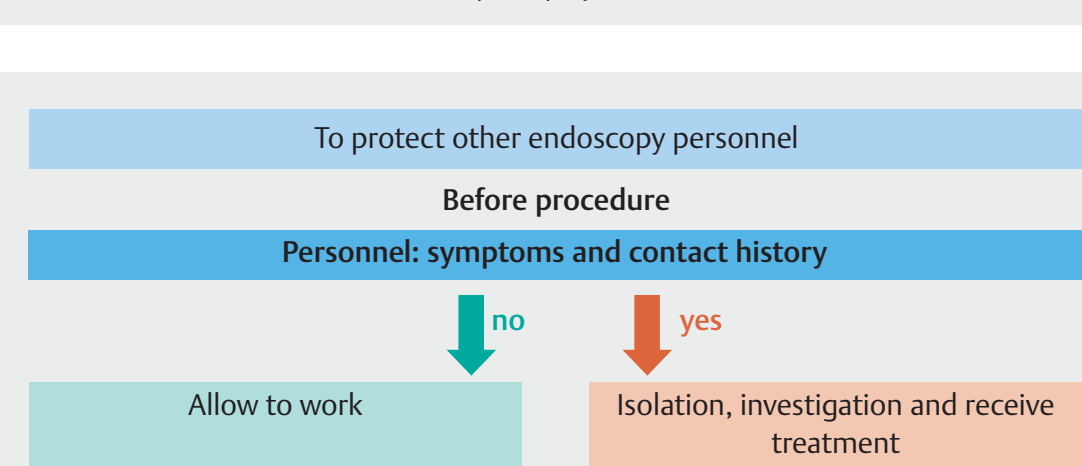

Staff protection

Fig. 2 Screening of endoscopy staff using symptoms and contact history.

During this epidemic crisis, all elective endoscopies have been canceled, as $\mathrm{Gl}$ endoscopy is considered high risk owing to contact with fecal matter, which may contain the virus. In addition, the hospital has used patient screening to place patients with suspected infection into the same designated wards. However, once the number of infected patients exceeded the normal hospital capacity, the Chinese government constructed special hospitals for COVID-19 patients [2]. During the TAGE GI Live sessions, invited faculty and all delegates were very im- pressed by the demonstrations and appreciated the efforts of the medical personnel in China. They stood together and mourned the loss of colleagues who had passed away from the virus infection.

Endoscopy_UCTN_Code_TTT_1AU_2AZ

Competing interests

The authors declare that they have no conflict of interest. 


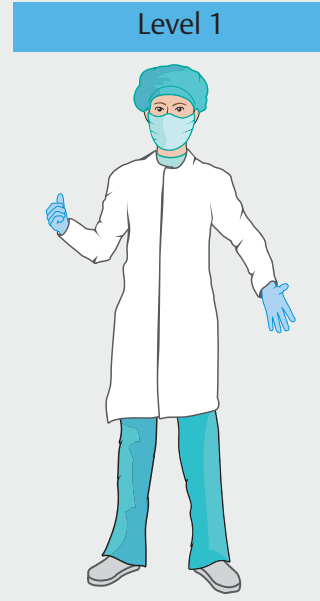

Medical hairnet Mask Gloves Medical coat

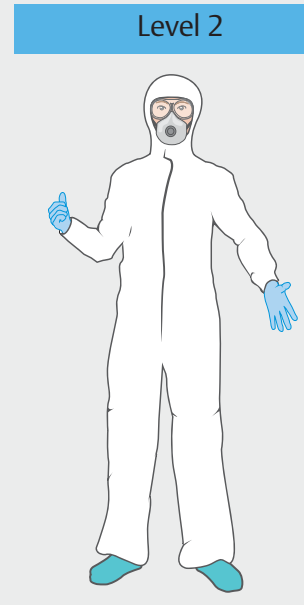

Medical hairnet

Mask

Gloves

Medical protective clothes Goggle/face shield

Shoe cover

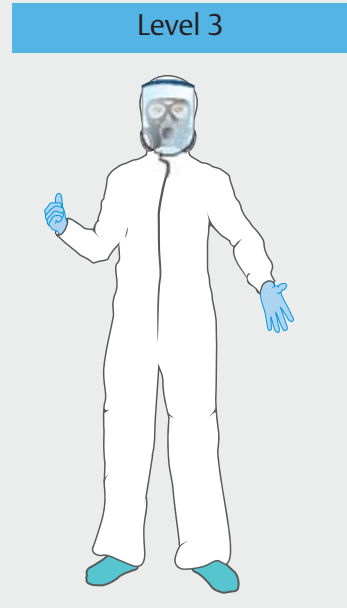

Medical hairnet

Mask

Double Gloves

Medical protective

clothes

Face shield

Shoe cover

Mask respirator

Fig. 3 The three levels of personal protective equipment recommended for protection of endoscopy staff. The level of protection depends on the infection risk of the patient.

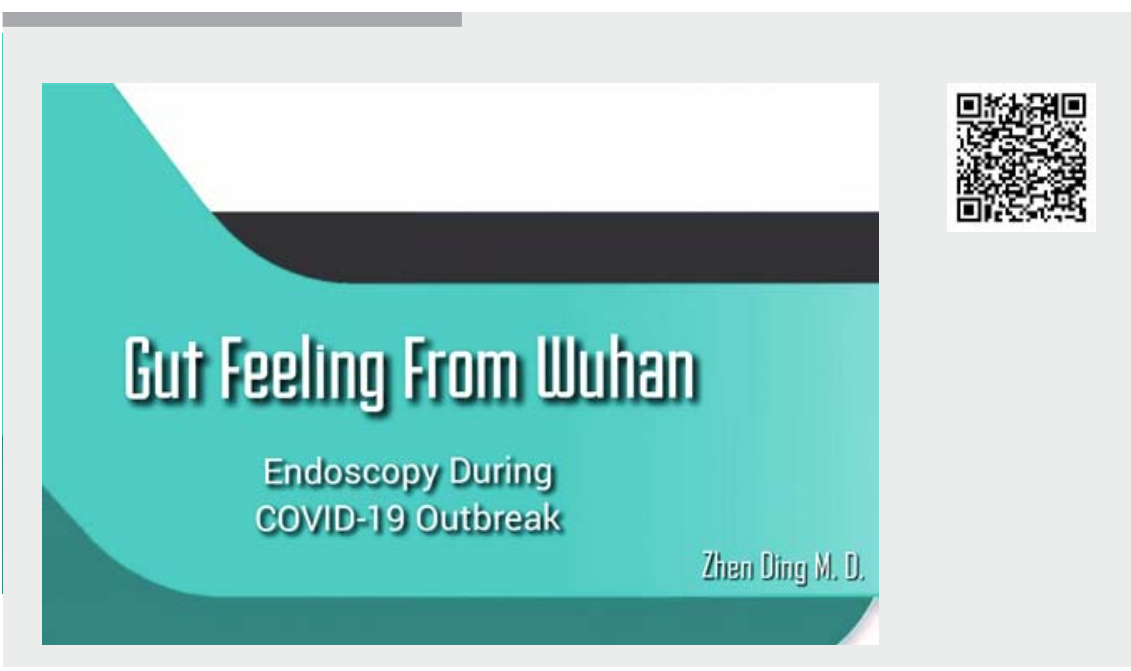

Video 1 This Video was a lecture from Wuhan during the 2020 annual meeting of the Thai Association for GI Endoscopy (TAGE). The session did not only allowed the endoscopy community to show its support for Chinese colleagues who have worked at the frontline, but TAGE and the GI (gastrointestinal) Live delegates learned about the protective measure taken by $\mathrm{Gl}$ endoscopy personnel during the outbreak.

The authors

Zhen Ding', Pradermchai Kongkam²,3,4, Thanawat Luangsukrerk ${ }^{2,3,4}$, Jun Liu', Yaping Cheng ${ }^{1}$, Thawee Ratanachu-ek ${ }^{3,5}$, Rungsun Rerknimitr $2,3,4$

1 Division of Gastroenterology, Union Hospital, Tongji Medical College, Huazhong University of Science and Technology, Wuhan, China

2 Gastrointestinal Endoscopy Excellence Center and Division of Gastroenterology, Department of Medicine, Faculty of Medicine, Chulalongkorn University and King Chulalongkorn Memorial Hospital, Thai Red Cross Society, Bangkok, Thailand

3 Thai Association for Gastrointestinal Endoscopy, Bangkok, Thailand

4 Pancreas Research Unit, Department of Medicine, Faculty of Medicine,

Chulalongkorn University, Bangkok, Thailand

5 Rajavithi Hospital, Ministry of Public Health, Bangkok, Thailand

\section{Corresponding author}

\section{Pradermchai Kongkam, MD}

Gastrointestinal Endoscopy Excellence Center and Division of Gastroenterology, 1873, GI Endoscopy Unit, 10th Floor Bhumisirimunkalanusorn Building, King Chulalongkorn Memorial Hospital, Rama IV Road, Pathumwan, Bangkok, 10330,

Thailand

Fax: +66-2-2564265

kongkam@hotmail.com

\section{References}

[1] Tablan OC, Anderson LJ, Besser R et al. Guidelines for preventing health-care-associated pneumonia, 2003: recommendations of CDC and the Healthcare Infection Control Practices Advisory Committee. MMWR Recomm Rep 2004; 53: 1-36

[2] Williams S. Coronavirus: how can China build a hospital so quickly? BBC News (31.01.2020); Available from (Accessed 28 February 2020): https://www.bbc.com/ news/world-asia-china-51245156

Bibliography

DOI https://doi.org/10.1055/a-1158-9016

Published online: 12.5.2020

Endoscopy 2020; 52: E261-E262

(c) Georg Thieme Verlag KG

Stuttgart · New York

ISSN 0013-726X 